\title{
BIOLOGIA REPRODUCTIVA E HISTORIA NATURAL DEL MERO (AGRIORNIS LIVIDUS) EN CHILE CENTRAL.
}

\author{
Manuel Marín \\ Natural History Museum of Los Angeles County, Section of Ornithology, 900 Exposition Boulevard, Los Angeles, \\ CA 90007, USA. Dirección actual: Casilla15 Melipilla, Chile, email: mma95@hotmail.com
}

\section{RESUMEN}

Se estudió la biología reproductiva del Mero (Agriornis lividus) principalmente en un terreno privado en la zona central de Chile. En Chile la especie es más abundante bajo los $1.700 \mathrm{~m}$, pero puede llegar a los $2.600 \mathrm{~m}$, prefiriendo laderas arbustivas de áreas áridas a semi-áridas, con arbustos espinosos, con algunos cactus, pero de preferencia áreas con Chaguales (Puya sp). En la zona central de Chile su época de nidificación se extiende desde principios de septiembre a finales de octubre con una nidada por temporada, si pierde su nidada al parecer no los reemplaza. Su nido era en forma de taza, pero muy voluminoso, la parte exterior fue construida con palitos gruesos y con otras ramillas. Su interior cubierto con una gruesa capa intermedia de musgos y luego forrado con musgos o fibras vegetales suaves y ocasionalmente pelos de mamíferos (e.g., conejos o caballos) y raramente algunas plumas de otras aves. La posición de los nidos variaba entre 1,5 a 2,5 m de altura. Había una tendencia de preferir plantas espinosas para nidificar principalmente en Chaguales (Puya chilensis) y algunas veces en cactus como Quiscos Echinopsis chiloensis. Su nidada varió entre dos a cinco huevos, siendo la mayoría $(47 \%)$ de tres huevos y $(41,2 \%)(n=17)$ de cuatro huevos y la mayoría de los huevos $(42 \%)$ era de la forma subelíptica. El color de los huevos era de un color base café cremoso con poco brillo casi opaco, con una gran variabilidad en el patrón de marcas. Aunque para algunos de los huevos el patrón de marcas era capirotado, con manchas irregulares, de un color rojizo, café-rojizo a negruzco, o café obscuro, estas marcas pueden ser: punteadas o moteadas. Se observó que solo la hembra incubaba. El período de incubación fue en promedio de 20 días (19-21) y los pichones abandonaron el nido entre los 18-20 días. Los pichones eran empollados día y noche hasta la edad de 7 a 8 días y luego hasta los 15 días solo parcialmente. El periodo $\mathrm{T}_{10-90}$ fue de 13 días y la constante de crecimiento $K=0,338$. El promedio de su masa corporal al eclosionar fue de $5,9 \mathrm{~g}$ y de su máxima masa fue $84,5 \mathrm{~g}$ a los 18 días de edad. La dieta de los pichones fue de un $82 \%$ entre lagartijas y orugas. Los adultos capturaban para los pichones principalmente presas del sotobosque $(70,4 \%)$, y el resto presas arbóreas. Los adultos en la época post reproductiva se alimentaban principalmente de presas del sotobosque, pero en su dieta adicionalmente incluían frutas como Calafate (Berberis microphylla) y Maitén (Maytenus boaria). La mayor mortalidad 32,5\% ocurrió en el estado de huevo. El éxito reproductivo total fue de 53,4\%. Es un migrante austral parcial, como en otros cazamoscas en algunos años parte de la población se queda a nidificar. A finales de marzo y principios de abril es cuando se han detectado pequeños grupos pasando por el área y moviéndose hacia el norte.

Palabras clave: Mero Agriornis lividus, biología reproductiva, dieta, crecimiento, historia natural, Tyrannidae, Chile.

\section{ABSTRACT}

Breeding biology and natural history of the Great Shrike-tyrant (Agriornis lividus) in central Chile. - The breeding biology of the Great Shrike-tyrant (Agriornis lividus) was mainly studied on private land in central Chile. Its main habitat is coastal arid to semi-arid hills in areas mainly with thorny bushes, cacti plants and with preference in areas with terrestrial bromeliads like Puya sp. Mostly below $1.700 \mathrm{~m}$, but locally it can reach up to $2.600 \mathrm{~m}$. Its breeding season extended from early September to late October with one brood per season and if eggs were lost there seems to be no replacement. The nest is typically cup shaped nest, a very bulky structure built with thick sticks, a mid-thick layer of mosses 
and lining was made either with only mosses or with soft vegetable items and sometimes added some mammalian hair depending on availability, e.g., horse or rabbit fur, and very rarely feathers of other bird species. Nest placement ranged between 1.5 to $2.5 \mathrm{~m}$ from the ground level. There was a tendency to prefer thorny plants mainly terrestrial bromeliad Puya chilensis, and sometimes a cactus Echinopsis chiloensis, for nest placement. Clutch size was from two to five eggs, but primarily three eggs (47\%) or four $(41.2 \%)(n=17)$ and most eggs $(42 \%)(n=50)$ were sub elliptical in shape. The eggs had as base color a matt to semi-glossy very pale brown or creamy brown with a large variability on the markings, ranging from almost no marks to a several irregular dots or blotches, very dark brown to reddish brown in coloration, sometimes forming a wreathed pattern. It was noted that only the female incubates. The average incubation period was 20 days (19-21) and the nestlings departed from the nest between 18-20 days. The nestlings were brooded full time until age 7-8 days and partially until 15 days of age. The $\mathrm{T}_{10-90}$ period was 13 days, and the growth constant was $K=0.338$. On average, the body mass of nestlings at hatching was $5.9 \mathrm{~g}$, and the maximum acquired was $84.5 \mathrm{~g}$ at 18 days of age. The observed nestling diet was $82 \%$ between lizards and caterpillars. The adults brought to the nest ground-dwelling prey items $(70,4 \%)$ and the rest were mainly arboreal but some aerial prey. Post-breeding adults also feed mostly on ground dwelling creatures and arboreal prey items, but also included some fruits like Calafate (Berberis microphylla) and Maitén (Maytenus boaria). Most mortality $32.5 \%$ occurred at the egg stage. The total reproductive success was $53.4 \%$. It is a partial austral migrant with some years part of the population stay to breed. Late March early April is when large numbers have been detected moving north through the area.

Key words: Great Shrike-tyrant, Agriornis lividus, breeding biology, diet, life histories, growth, Tyrannidae, Chile.

\section{INTRODUCCIÓN}

En la familia tyrannidae hay cinco especies agrupadas en el género Agriornis, que es un grupo en que todos sus miembros son de cuerpo grande y bastante distintivos. Tienen picos grandes y ganchudos que son usados para capturar presas de gran tamaño inclusive pequeños vertebrados. Son de comportamiento bastante discreto, casi silenciosos, sus sonidos pasan fácilmente desapercibidos y algunos son casi inaudibles; son pobremente conocidos, aparte de su distribución generalizada (e.g., vease Fitzpatrick, 2004). Son de áreas abiertas de ambientes semi áridos a áridos con pequeños arbustos a lo largo de la Cordillera de Los Andes y Patagonia.

El Mero (Agriornis lividus) una especie restringida al extremo sur/suroeste de Sudamérica y es uno de los cazamoscas de tamaño grande, siendo el más grande que hay en Chile, y si puede haber dimorfismo sexual, pero no un dicromatismo sexual marcado. Adicionalmente hay una diferencia de tamaño entre los individuos de la zona central y los de la zona Austral. Habita lugares semiáridos: en zonas de dunas costeras con vegetación, en quebradas y laderas con cactus o chaguales (Puya sp.) y en áreas abiertas de vegetación achaparrada. Se distribuye desde la Región de Atacama hasta la Región del Maule y luego por la zona cordillerana, incluyendo el oeste de Argentina (desde Neuquén al sur) llegando a la zona norte de la Isla de Tierra del Fuego (Goodall et al. 1957, Humphrey et al. 1971). Su rango altitudinal en la zona central de Chile es principalmente bajo los $1.700 \mathrm{~m}$, a mayor altura y más al norte es reemplazado por el Mero Gaucho Agriornis montanus. Es un migrante austral parcial, y una especie poco común en todo su rango, pero siendo más abundante en la zona centro sur de Chile. Información sobre la biología reproductiva del género Agriornis es básicamente nula, para las cinco especies del género, no se conoce más allá de la descripción del nido y de sus huevos. El objetivo de este estudio fue resumir lo conocido y lo investigado sobre algunos aspectos de distribución, incubación, crecimiento, dieta, mortalidad y otros aspectos de la historia natural del Mero Agriornis lividus. 


\section{ÁREA DE ESTUDIO Y MÉTODOS}

Información general fue tomada en diferentes puntos del país, pero el área principal de estudio fue en el sector de San Manuel, prov. Melipilla, Región Metropolitana ( $160 \mathrm{~m}, 33^{\circ} 46^{`} \mathrm{~S} 71^{\circ} 18^{`} \mathrm{~W}$ ), al término este de la planicie costera. El área general es de un clima mediterráneo con un invierno corto y de poco frío y un verano seco y caluroso, con un promedio de precipitación anual de $464 \mathrm{~mm}$ (Cunnill 1970). Las observaciones se concentraron en un área de unas 100-150 ha, de cerros cubiertos con vegetación nativa, de crecimiento secundario. Los árboles y arbustos dominantes en el área, principalmente las laderas lado sur o de sombría, son el Trevo/Tebo (Trevoa trinervis), el Espino (Vachellia cavem) y el Quillay (Quillaja saponaria), secundado por especies como el Molle (Schinus latifolius), el Maitén (Maytenus boaria) y el Bollén (Kageneckia oblonga). Adicionalmente, en las zonas de laderas de exposición norte o de solana comúnmente crecen Chaguales Puya chilensis, Chagualillos Puya coerulea, y Quiscos Echinopsis chiloensis. En el área de estudio, el Mero no nidifica todos los años, en años de lluvias normales (464 mm) a lluviosos puede ser abundante mientras que en años secos a muy secos o cuando las lluvias son menores que el promedio anual, su número es reducido a casi inexistente. Su tamaño poblacional aumenta notoriamente entre los meses de marzo/abril y luego en agosto/septiembre durante sus movimientos migratorios (ver también abajo).

Durante los periodos reproductivos del 2008 al 2017 se tomaron datos de 18 nidos, se les hizo seguimiento de desarrollo a 29 polluelos de 12 nidos: a 20 desde la eclosión = día cero, hasta salir exitosamente del nido, seis desde su eclosión hasta su desaparición por depredación y tres que se encontraron ya eclosionados. La mayoría de los pichones fueron medidos diariamente, pero en unos pocos casos a intervalos de dos días dependiendo de la disponibilidad de tiempo. El 80\% de los pichones fueron medidos y pesados en la mañana antes de las 12:00 h y el resto se midió y pesó en la tarde después de las 17:00 h. Si los pichones eclosionaban por la mañana y se llegaban a medir por la tarde, o viceversa, se les incremento/o resto la edad en medio día, respectivamente. Esto último fue tenido en cuenta debido a que en un periodo de 6 a 8 hrs, dependiendo de su alimentación, puede generar una gran diferencia, variando entre un 12 al 35\% en su masa corporal, en particular en las edades intermedias donde el crecimiento es más rápido (observ. pers.). Para la masa corporal de huevos y pichones se ocuparon balanzas tipo Pesolas AVINET (a 1,0 g) de 10,30 , y $100 \mathrm{~g}$. Para medir ala y cola, se uso una regla milimétrica (a $1,0 \mathrm{~mm}$ ) y para medir culmen expuesto y tarso, se utilizó un calibrador milimétrico (a $0,1 \mathrm{~mm}$ ), siguiendo la forma estandarizada de Baldwin et al. (1931). Fueron calculadas, el tiempo entre el 10-90\% del crecimiento o el periodo $\left(\mathrm{T}_{10-90}\right.$; Case 1978) y la constante de crecimiento $K$ (Ricklefs 1976, 1983). Los periodos de incubación se tomaron desde la postura del segundo huevo hasta la eclosión del primer huevo. Para las formas de los huevos se sigue la forma estandarizada de Preston, (Palmer 1962:13). Para la masa corporal y medidas de adultos; Tabla 1, se usaron medidas descritas en la literatura y especímenes de museo solo de la zona central de Chile. También se usaron especímenes de museo para datos de dieta, elevación u otra información relevante. Especímenes depositados en American Museum of Natural History, New York, EE.UU., ex Colección Francisco Behn (CFB), Zapallar, Chile, Los Angeles County Museum of Natural History, Los Angeles, EE. UU., Museum of Comparative Zoology, Harvard University (MCZ), Cambridge, EE.UU., Museo Nacional de Historia Natural, Santiago, Chile y la Western Foundation of Vertebrate Zoology, Camarillo, EE. UU.

\section{RESULTADOS Y DISCUSIÓN}

\section{Habitat}

El Mero habita zonas áridas a semi áridas, áreas como: zonas de matorrales, zonas de dunas con vegetación achaparrada, pampas con algo de arbustos, en espinales en bosques de secano semi abiertos, quebradas y laderas con cactáceas y puyas, no entra en zonas boscosas siempreverdes, ni zonas muy 
pobladas (Albert 1899, Goodall et al. 1957, Vuilleumier 1994, observ. pers.). No es de zonas boscosas y en acuerdo con Vuilleumier (1994) esta especie no vive en áreas muy abiertas o de pastizales, como básico requiere zonas con arbustos. Fjelså y Krabbe (1990) describen su hábitat en áreas abiertas, semiáridas con Mata Verde o Romerillo Chiliotrichium sp., cactus, y bromelias, áreas transicionales de Nothofagus. En la zona de estudio se le encuentra en zonas semiabiertas, principalmente en las laderas de solana con cactus Quiscos Echinopsis chiloensis, Chaguales Puya chilensis y/o Chagualillos Eryngium paniculatum. En invierno se le puede encontrar en áreas de plantaciones de almendros (Prunus dulcis) y similares. No se le encuentra en áreas de cultivo ni áreas con mucha vegetación siempre verde ya sea natural o plantaciones. Requiere áreas donde tenga visibilidad para posarse en alguna percha de acecho y de ahí atacar a su presa, similar a un halcón.

\section{Distribución}

En Sudamérica, el Mero tiene una distribución angosta pero larga principalmente dentro de Chile, pero también habita en una larga y angosta franja del área andina del centro/sur oeste de argentina. En Chile, en su extremo norte solo se le encuentra por la zona costera y su límite en invierno solo alcanza hasta La Aguada/Quebrada del León (27으) (Gigoux 1928b, observ. pers.). Curiosamente, no fue mencionada por Millie (1938), en su publicación sobre las aves del Valle del Huasco, donde debe ser solo transitoria, la he observado en las partes costeras del Valle en los meses de abril y mayo. Hellmayr (1932) lo da desde Copiapó hasta Curicó. Desde la Región de Coquimbo, zona de La Serena/Coquimbo hacia el sur, su distribución va en dos secciones cortadas por las partes altas de la cordillera de la costa la primera va por la costa hasta las laderas oeste de la cordillera de la costa y la segunda por los contrafuertes cordilleranos. Para la subespecie nominal, por la zona cordillerana, hasta la provincia del Biobío, Región del Biobío, en áreas de vegetación achaparrada y escaza, y en áreas de pampa. La especie ha sido capturada en el mes de febrero en el área de la Laguna del Laja, prov. Biobío. Aunque, Goodall et al. (1957) y Philippi (1964) da su distribución sur llegando a la zona norte de la Región de los Ríos, pero ahí es solo un visitante temporal en dicha área. Por la zona costera para la subespecie nominal su área reproductiva es más acotada, por el norte va desde la Región de Coquimbo y su límite sur de reproducción no está bien definido, pero Hellmayr (1932) lo da hasta Curicó en la Región del Maule. Aunque, podría ser algo más al sur hasta la provincia de Nuble, en la Región del Biobío.

Autores como Hellmayr (1932), Humphrey et al. (1970) y Ridgely y Tudor (1994) dan su distribución desde la parte sur de la ex provincia de Llanquihue, Río Ñirehuau = Ñihuerao (hoy es la Región de Aisén, prov. de Coihaique) hasta la parte norte de la Isla grande de Tierra del Fuego, prov. Tierra del Fuego. Considero que su límite norte es más al norte de lo mencionado por dichos autores. Su distribución norte seria desde las zonas cordilleranas con ambiente de estepas y arbustivas al menos desde la Región de la Araucanía hacia el sur, inclusive puede ser por la zona cordillerana algo más al norte. En todo caso desde la Región de la Araucanía hacia el sur por la cordillera la subespecie nominal sería reemplazada por la subespecie austral Agriornis lividus fortis, la cual tiene la cola y alas más largas y una mayor masa corporal que oscila entre los 95 a 115 g versus 73 a 93 g, (datos de masa de especímenes de museo, ver agradecimientos). Hellmayr (1932) menciona la captura de juveniles en el mes de febrero en el área de Lonquimay. Adicionalmente, hay especímenes capturados en el área de Lonquimay por Francisco Behn $(\mathrm{CBF})$ en el mes de enero con testículos en vía de receso, pero relativamente grandes ( 7 y $8 \mathrm{~mm}$ de largo). Los juveniles capturados y los especímenes con las gónadas grandes, sugiere que se reproduce en el área. El área de Lonquimay es un enclave de estepa patagónica muy similar al área del Río Ñihuerao, prov. de Aisén, lugar que fue dado como su límite norte por varios autores (ver arriba). A la subespecie austral se le puede encontrar en zonas cordilleranas en áreas de pampa y de escaza vegetación desde la región de la Araucanía (al menos desde el área de Lonquimay $38^{\circ} 30^{\prime} \mathrm{S}$ ), en la zona costera y valles centrales es solo transitoria, (ver también abajo) hasta la parte norte de la isla de Tierra del Fuego $\left(53^{\circ} \mathrm{S}\right)$. Para la subespecie 
austral por el este de la cordillera por Argentina lo dan desde Neuquén $\left(c a .38^{\circ} \mathrm{S}\right)$ hasta la Isla de Tierra del Fuego (e.g., Zotta 1944, Kovacs et al. 2005). En áreas costeras como Arauco y Concepción (Lane 1897, Reed 1904) y áreas de la depresión central como Angol y Perquenco (NE Temuco) (Bullock 1929, Kuroda 1933) parece ser solo un visitante transitorio en invierno (ver también abajo).

Su distribución altitudinal varia en diferentes autores, por ejemplo: Barros (1921) para la Región de Valparaíso lo da hasta los $1.800 \mathrm{~m}$. Hellmayr (1932) lo da para el país desde la zona costera a los $1.800 \mathrm{~m}$. Housse (1945) lo da entre los 2.000 a $3.300 \mathrm{~m}$ [aunque poco posible] indica que es un migrante altitudinal y en invierno baja a los valles. Luego Goodall et al. (1951) y Barros (1961) indican que solo llega hasta los 2.000 m. Johnson (1967) y Fjeldså y Krabbe (1990) lo dan hasta los 1.800 m y Ridgely y Tudor (1994) lo sitúan como mayoritariamente bajo los 1.500 m. R.A. Philippi, colecto un individuo (MCZ \# 287413) el 30 de abril de 1939 en el Valle de los Leones, (32 44'S) 2.500 m., prov. Los Andes (por la fecha podría tratarse de un migrante austral) y más recientemente, el 15 de octubre del 2020, fue avistado en la Parva (33 $\left.18^{\prime} \mathrm{S}\right)$ a $2.600 \mathrm{~m}$, prov. Santiago (Rodrigo González, información sin publicar). Sugiriendo de que podría reproducirse a esa altura. Mis observaciones lo dan reproduciéndose, al menos hasta los 1.700 m. (cordillera de la Costa, Cerro Matancilla) y tal vez puede reproducirse a mayor altura. Aunque, en mis observaciones de varios años y en diferentes épocas del año y lugares no lo he encontrado más allá de los $1.700 \mathrm{~m}$. Para la zona central de Chile coincido con Ridgely y Tudor (1994) que es más abundante bajo los $1.500 \mathrm{~m}$. Adicionalmente, a mayor altura, sobre los $2.000 \mathrm{~m}$ en general es reemplazado por el Mero Cordillerano o Mero Gaucho Agriornis montana. Aunque en la zona central de Chile localmente entre los 1.800 y los $2.600 \mathrm{~m}$ es una zona de traslapo donde ambas especies de Agriornis, son simpátricas. No se sabe si ambas especies nidifican en esa zona de sobreposición en la zona central o solo coinciden en ciertos periodos del año. Más al sur la zona de traslapo es más amplia y en la zona austral es prácticamente total. Lo cual sería un área interesante de explorar en detalle.

\section{Fenología Reproductiva}

La información publicada sobre su época de nidificación está basada en poca información. Germain (1860) indica que su postura es entre octubre y noviembre. Luego Goodall et al. (1957) indican que en la región de la costa nidifican en octubre y al interior en la precordillera no antes de noviembre. Barros (1961) solo menciona que empieza anidar muy temprano ya que el día 29 de agosto encontró un nido con tres huevos, en la zona de Melipilla. Otros autores subsecuentes e.g., Johnson (1967), Fjeldså y Krabbe (1990), solo repiten lo de Goodall et al. (1957). El área de estudio se le puede considerar como zona costera y de 14 nidos su nidificación fue entre septiembre y octubre. Se encontró que cinco nidos $(35,7 \%)$ comenzaron su incubación en la primera quincena de septiembre y la gran mayoría nueve nidos $(64,2 \%)$ comenzaron su incubación en la primera quincena de octubre. La fecha más temprana de incubación fue el 1 de septiembre 2010 y la más tardía el 14 de octubre 2017. Para la zona cordillerana información de siete nidos [especímenes de museo y Housse (1945)], encontrados en la zona cordillerana la postura fue en el mes de noviembre. Los resultados coinciden con Goodall et al. (1957) en que si hay una diferencia altitudinal en el ciclo de reproducción entre las poblaciones cordilleranas y las que viven debajo de los $1.000 \mathrm{~m}$ de altura o poblaciones costeras. La diferencia entre ambas poblaciones es que en las zonas costeras su ciclo comienza por lo menos un mes antes de lo señalado por diferentes autores (ver arriba). Adicionalmente, el encuentro de Barros (1961) y los de este estudio indican, que en algunos años fácilmente podría comenzar a finales de agosto. Esto probablemente dependiendo de las lluvias. La cantidad de individuos que nidifica en el área varía cada temporada, en años muy secos es raro a nulo y en años lluviosos puede ser una especie abundante. Para la zona austral, aunque no hay datos concretos debería ser similar a lo observado en las zonas cordilleranas de la zona central. 


\section{Territorios, Nido y Posición}

Todos los nidos encontrados en este estudio eran en pendiente de cerro y fácilmente se podía observar los desplazamientos de los adultos. En particular del macho que tenía la tendencia de posarse en perchas en altura y así teniendo una gran visibilidad del territorio. De los territorios que logré obtener medidas aproximadas uno fue de unas 10 hectáreas, y un segundo fue de unas 8 hectáreas. Eran territorios extensos y los nidos estaban todos localizados en quebradas de solana, ningún nido fue localizado en quebradas de umbría. Es decir, todos con algo de cara en dirección norte. Adicionalmente, había unos 400$500 \mathrm{~m}$ como distancia mínima entre nidos. Dos territorios fueron mantenidos por un mínimo de tres años y uno por un mínimo de cinco años. En tres ocasiones los nidos fueron construidos en el mismo Chagual al año subsecuente, pero este fue construido entre 50 a $100 \mathrm{~cm}$ de distancia del nido que uso el año anterior.

Hay pocas descripciones del nido de esta especie. Germain (1860) indica que es un nido mal hecho y localizado en cavidades en taludes, por cierto, algo poco posible (ver abajo) y lo más probable es que confundió un nido de Mero Gaucho (Agriornis montana) con esta especie. Housse (1945) describe un nido "en pleno matorral a un metro del suelo muy escondido en lo más tupido de las ramitas". Goodall et al . (1957) lo describen como un nido grande y abultado, hecho de pasto y algunos palitos en su exterior forrado con lana de oveja. Adicionalmente indican que coloca su nido en arbustos tupidos, quiscos o arboles chicos de follaje espeso. Barros (1961) lo describe que fue construido entre las arqueadas y espinudas hojas de un Chagual. En el área de estudio de 15 nidos observados 13 fueron en Chaguales construidos en la base de las arqueadas y espinudas hojas (ver Figura 1) y dos en las bifurcaciones de los brazos de Quiscos. Los nidos eran abultados, tipo taza y muy variables en el tamaño exterior, construidos con gran cantidad de palitos secos y gruesos en su base y exterior, luego una gruesa capa de musgos, pequeñas raíces y forrado con musgos, algunos nidos contenían un par de plumas, probablemente del propio adulto, otros pelos de conejo o de caballo, pero en la gran mayoría el forro era solo musgos y algunas raíces suaves (Fig. 1). El diámetro interno fue en promedio $111 \mathrm{~mm}(\mathrm{DE} \pm 5,4$, rango $=105-120 \mathrm{~mm}, \mathrm{n}=13)$ y de profundidad de 68 $\mathrm{mm}(\mathrm{DE} \pm 4,6$, rango $=65-80 \mathrm{~mm}, \mathrm{n}=13)$. Los nidos estaban construidos a baja altura, el más bajo a $1,5 \mathrm{~m}$ y el más alto a $2,5 \mathrm{~m}$, en promedio $1,8 \mathrm{~m}(\mathrm{DE} \pm 0,38)$. El encuentro de Barros (1961) fue lo más cercano a lo encontrado en el área de estudio. Los nidos descritos por Germain (1860) y Housse (1945) se considera que están algo fuera de lugar. La duración de la construcción del nido desde los primeros palitos hasta el final, solo se pudo conprobar con precisión para dos nidos y en ambos fue de nueve días. Otros nidos solo se pudieron observar parcialmente. Sin embargo, se observó que solo uno de los adultos, presumiblemente la hembra, construía el nido mientras que el segundo se posaba en una percha en altura, con gran visibilidad, haciendo de guardia. Al parecer en la familia Tyrannidae, solo la hembra es la que toma el rol activo en la construcción del nido sin ayuda de su pareja (Fitzpatrick, 2004), lo encontrado en esta investigación concuerda con eso.

\section{Huevos e Incubación}

La nidada fue mencionada como de dos a cuatro huevos por Germain (1860). Housse (1945) solo menciona dos nidadas una de dos y una de tres huevos. Goodall et al. (1957) indican que las nidadas son de dos y raramente cuatro huevos. Johnson (1967) menciona que las nidadas son generalmente de tres, a veces de dos y ocasionalmente de cuatro huevos. Se encontró que de 17 nidadas: una fue de dos (5,8\%), ocho de tres $(47 \%)$, siete de cuatro $(41,2 \%)$ y una de cinco $(5,8 \%)$. Indicando que la nidada es entre tres y cuatro huevos y ocasionalmente dos o cinco, lo cual poco coincide con lo descrito en la literatura. Housse (1945) al parecer fue el primero en describir los huevos. Detalla para dos nidadas diferentes, la primera como "de un verde subido con manchitas castañas y algo lilas", lo cual no se ajusta a los huevos observados de esta especie. Para la segunda nidada indica que sus huevos "son blancos con algunas pintas castañas" la segunda nidada si se ajusta a esta especie. Pero, indica que el color de los huevos varía mucho, lo cual, por lo observado, Housse no está en lo correcto. Su descripción de la primera nidada es sin duda de otra especie. 


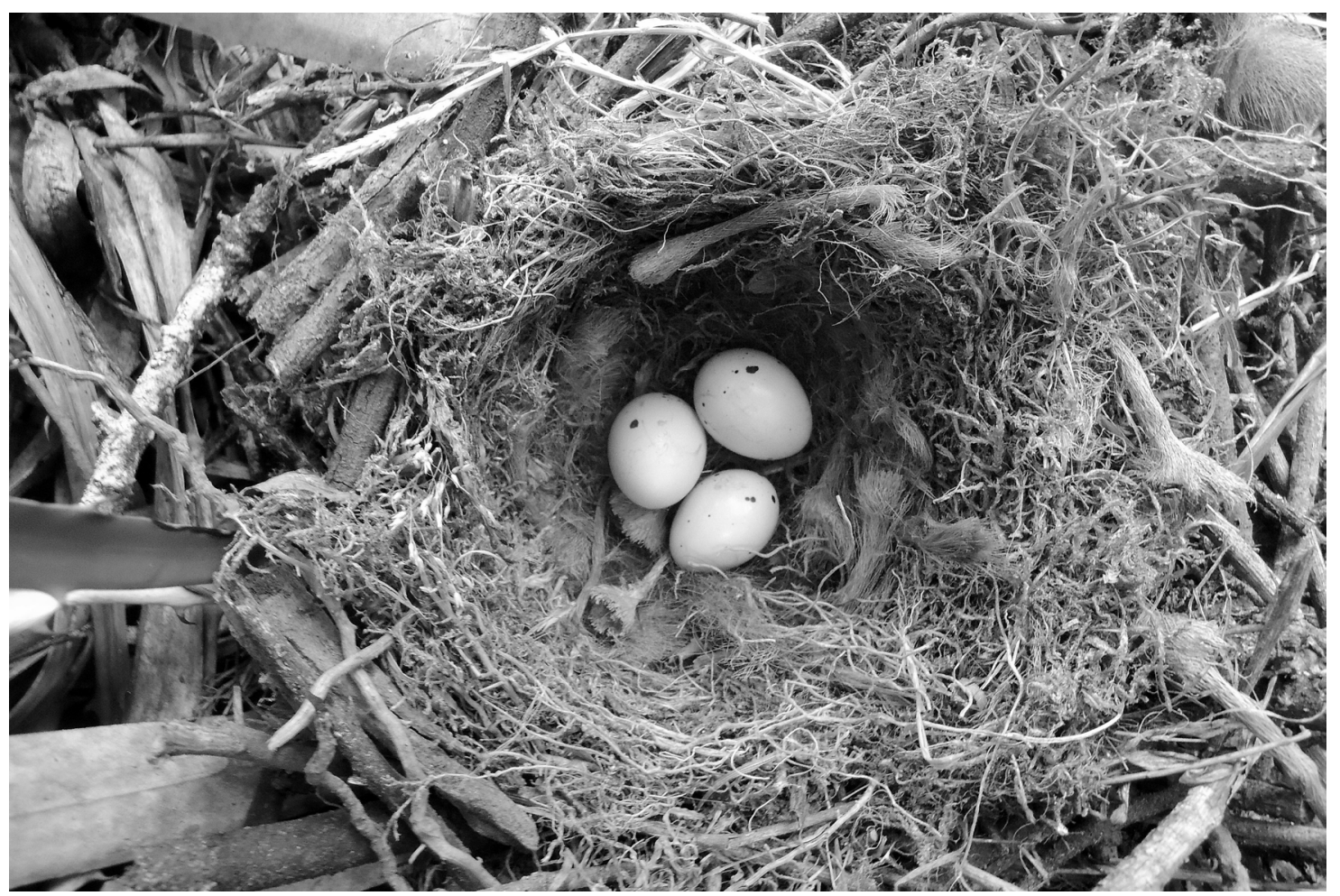

Figura 1.Nido de Mero (Agriornis lividus) construido en un Chagual (Puya chilensis) en el sector San Manuel, Melipilla, Chile. (Foto: M. Marín).

Goodall et al. (1957) indican que son similares a otros cazamoscas, pero se distinguen por el color de fondo que es marcadamente más cremoso. Barros (1961) indica que sus huevos "son blancos y presentan pintitas y puntos café rojizos u obscuros en el polo grueso y parte ancha". Todos los huevos encontrados eran de un color café pálido semi-opaco (o un color café con leche) manchado en forma irregular, principalmente en el polo más ancho, con manchas de color café obscuro a café rojizo (ver también Fig. 1). En cuanto a la coloración la descripción de Goodall et al. (1957) se asemeja más a lo encontrado en este estudio. La forma de los huevos variaba desde: subelíptica corta 4 (8\%); subelíptica 21 (42\%); subelíptica larga 2 (4\%); oval $9(18 \%)$; y oval corta, $14(28 \%)(n=50)$, con su gran mayoría siendo subelíptica. En cuanto al tamaño de los huevos no es tan diferente a lo presentado por diferentes autores, para las medidas de los huevos ver Tabla 2. Solo tenían una postura por temporada en caso de pérdida por depredación u otro motivo no remplazaban los huevos.

Se pudo confirmar que el adulto comenzaba a incubar no antes de la postura del segundo huevo. Adicionalmente, esto se reflejaba en que los dos primeros pichones eclosionaban a pocas horas de diferencia mientras el tercer, cuarto o quinto huevo eclosionaba con uno a dos días de diferencia. El periodo de incubación se computó desde la postura del segundo huevo hasta que eclosionó el primer pichón y este fue de $20 \pm 0,63$ días (rango=19-21, $\mathrm{n}=6$ ). Sobre su incubación se observó que solo uno de los adultos incubaba, presumiblemente la hembra, el segundo individuo que presumiblemente era el macho pasaba posado en perchas que actuaban de miradores. El supuesto macho al acercarse uno al nido emitía una serie de sonidos de una frecuencia alta poco discernible al oído. Inicialmente, solo al estar muy cerca del nido era más vocifero, hasta que después de algunos días se habituaban a mi presencia. De acuerdo con Fitzpatrick (2004) de los miembros de la familia Tyrannidae solo la hembra incuba, el encuentro de este 
estudio corrobora que solo un miembro, presumiblemente la hembra construye el nido e incuba los huevos. Al eclosionar, la hembra empollaba los pichones continuamente hasta la edad de siete a ocho días y entre los ocho a 15 días solo los empollaba parcialmente.

\section{Desarrollo de los polluelos}

Al eclosionar la hembra tomaba las cáscaras de los huevos y las llevaba y botaba a unos 15 a 20 $\mathrm{m}$ del nido. Los pichones al eclosionar son típicamente altriciales con los ojos cerrados, con una visible línea de ojo, el culmen era de color anaranjado, con la parte distal de un color grisáceo y con un débil diente de huevo. El culmen, a los 3-4 días, era más notorio su obscurecimiento y pasaba a un tono amarillo grisáceo y a los siete días cambiaba a un tono negro amarillento, para luego a los 17 días, el culmen ya era negruzco con la punta algo amarillenta. La disolución del diente de huevo fue una de las características que más varió entre individuos y fue entre los 13 a 18 días de edad. El revestimiento interior de la boca era de un color amarillo anaranjado brillante, con los bordes exteriores más amarillos, los rebordes de un color blanco amarilloso. El cuerpo, piernas y patas de un color rosado anaranjado, con las uñas grisáceas. Las piernas y pies se tornaban grisáceas a los 10-11 días de edad y antes de salir del nido ya eran negruzcas con las uñas negras. El cuerpo al eclosionar tenía plumón de color negruzco, bastante denso en particular en el dorso y nuca. Entre los 4 y 5 días se abría la línea del ojo para tenerlos totalmente abiertos a los 9-10 días. El promedio de su masa corporal al eclosionar fue de 5,9 $\mathrm{g}(\mathrm{DE}=0,52$, rango $=5,0-6,7 \mathrm{~g}, \mathrm{n}=20)(7,1$ $\%$ del tamaño del adulto) su masa aumentaba diariamente en forma linear hasta la edad de 13 días donde el incremento diario disminuía levemente. Algunos individuos lograban obtener la masa del adulto y la máxima masa corporal adquirida por los pichones antes de salir del nido, fue de 84,5 g a los 18 días (102,5 $\%$ del tamaño del adulto). La masa mínima al salir del nido fue de 69,5 g (84,3\% del tamaño del adulto) (Fig. 2A, para medidas del adulto ver Tabla 1). Estas diferencias pueden ser por una diferencia morfológica entre géneros, daba la impresión de que las hembras eran más pequeñas, lo cual no se pudo analizar por falta de material. El periodo $\mathrm{T}_{10-90}$ fue de 13 días y la constante de crecimiento $K=0,338$. No se encontró información comparativa sobre crecimiento y desarrollo para alguna especie de tamaño similar, pero Starck y Ricklefs (1998) en un resumen de parámetros de crecimiento dan un rango entre 10 y 15 días para el periodo $\mathrm{T}_{10-90}$ para miembros pequeños (de máxima masa 38,3 g) de la familia tyrannidae, pero el Mero estaría dentro del rango de dicha familia.

Al eclosionar el tamaño promedio de las alas de los pichones fue de $8,7 \mathrm{~mm}(\mathrm{DE}=0,85) 6,6 \%$ del tamaño del adulto. La máxima medida del ala antes de salir del nido fue de 91,5 $\mathrm{mm}$ a los 19 días 63,3\% del tamaño del adulto (Fig. 2B). Los cañones de la cola comenzaron a emerger a los cinco días y el máximo crecimiento de la cola dentro del nido fue de 55,9 mm a los 20 días, 50,8\% del tamaño del adulto (Fig. 2C). Al eclosionar el tamaño promedio de él tarso fue de $9,3 \mathrm{~mm}(\mathrm{DE}=0,58) 24,6 \%$ del tamaño del adulto y a los 12-13 días adquirieron el 100\% del tamaño del adulto (Fig. 2D). Siendo el tarso la única medida que adquirió el tamaño del adulto dentro del nido. El promedio del culmen al eclosionar fue de 5,6 $\mathrm{mm}(\mathrm{DE}=$ 0,78) 19,8\% del tamaño del adulto y el máximo antes de salir del nido fue de $21,9 \mathrm{~mm}$ a los 20 días 77,6\% del tamaño del adulto (Fig. 2E) (para parámetros de desarrollo ver Tabla 3).

\section{Dieta}

El Mero principalmente forrajeaba en el suelo donde capturaba sus presas como lagartijas, insectos, y arácnidos. Pero también se le observo mucho capturando presas desde perchas de acecho. Dos tipos de perchas de acecho: percha baja hasta $1 \mathrm{~m}$, donde se movía desde la percha al suelo a capturar presas como coleópteros terrestres, arácnidos, lagartijas, ortópteros, o desde perchas más altas variando de 2 a $10 \mathrm{~m}$, donde volaba en captura de presas voladoras como odonatos, lepidópteros o cualquier presa voladora que pueda ser capturada en forma oportunista, inclusive aves pequeñas (e.g, Martinez del Río 1992, Ridgely y Tudor 1994, Nuñez 1995). A cualquier presa una vez capturada la golpeaba contra alguna piedra, rama 

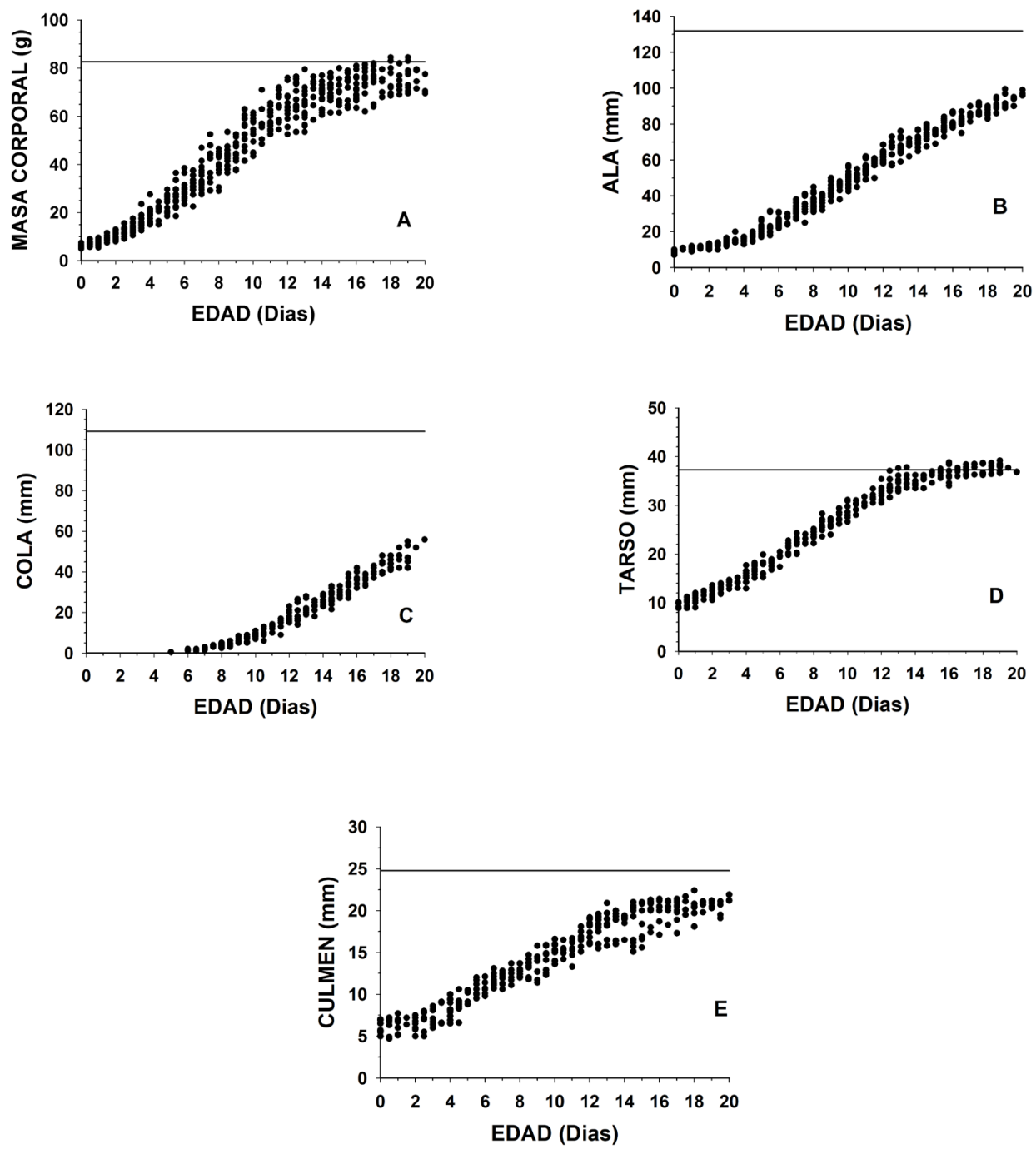

Figura 2. Curvas de crecimiento de cinco parámetros para los pichones de Mero (Agriornis lividus) tomadas en el sector San Manuel, Melipilla, Chile. A) masa corporal, B) ala, C) cola, D) tarso y E) culmen. La línea horizontal solida en la parte superior de cada grafico representa el tamaño del adulto (ver Tabla 1 y texto).

o el suelo mismo para asegurar su muerte antes de ser consumida. En la literatura solo se han descrito observaciones sobre lo que el adulto captura o se ha comido, que no necesariamente pudiera ser lo mismo que alimenta a los pichones. En el presente trabajo describo ambas circunstancias por separado.

A) Dieta de los adultos: Todas las menciones de la dieta del Mero han sido descritas en base a observaciones de los adultos o contenidos estomacales. Su dieta ha sido referida como un ave insectívora por Fraser (1843) y Reed (1924). Reed (1924) indica gran cantidad de insectos que encontró en el estómago de un individuo colectado en Lampa el 4 de mayo de 1924, entre los que pudo identificar incluía Bupréstidos, Bostríquidos, Tenebriónidos y larvas de Elatéridos. Housse (1945) indica correctamente que mucho le gustan las lagartijas, observó uno con un pequeño roedor, otros que habían consumido larvas de insectos xilófagos, larvas en general, hormigas, hemípteros, ortópteros, y pequeños batracios, es decir cualquier organismo comestible 
Tabla 1. Masa corporal y medidas morfológicas de Agriornis lividus de Chile central, basadas en especímenes de museo.

\begin{tabular}{lccc}
\hline Característica & MediaPro & DE & n \\
\hline Masa $(\mathrm{g})$ & 82,4 & 6,59 & 11 \\
Ala $(\mathrm{mm})$ & 132,0 & 3,79 & 16 \\
Cola $(\mathrm{mm})$ & 109,9 & 4,55 & 16 \\
Tarso $(\mathrm{mm})$ & 37,6 & 0,89 & 16 \\
Culmen $(\mathrm{mm})$ & 28,2 & 1,78 & 16 \\
\hline
\end{tabular}

Tabla 2. Dimensiones y masa de los huevos del Mero (Agriornis lividus) de Chile central estudio

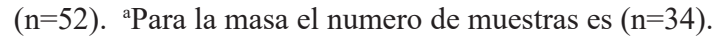

\begin{tabular}{ccccc}
\hline Característica & Media & DE & EE & Rango \\
\hline Largo (mm) & 29,43 & 1,15 & 0,16 & $27,2-32,0$ \\
Ancho (mm) & 21,83 & 0,63 & 0,08 & $20,3-23,2$ \\
Masa a (g) & 7,6 & 0,55 & 0,09 & $6,0-8,5$ \\
\hline
\end{tabular}

encontrado en el suelo. Housse (1945) y Barros (1961) adicionalmente indican que consume las frutas de Maitén Maytenus boaria, lo cual también he observado, pero solo entre los meses de marzo y mayo. Goodall et al. (1957) lo mencionan que se alimenta en parte de insectos, lagartijas, sapos, pequeños roedores e inclusive huevos. Etiquetas de especímenes de museo indican como cosa más común encontradas en sus estómagos han sido: orugas, coleópteros, ortópteros, pero también un par de etiquetas indica restos de frutas como Calafate Berberis microphylla o Maitén. Entre las presas observadas capturadas por los adultos en el periodo post reproductivo en el área de estudio incluía, caracoles (Helix aspersa), lagartijas (Squamata), arañas (Arachnida), orugas y hespéridos (Lepidóptera), chicharras (Cicadidae), insectos varios (Coleoptera: Carabidae, Tenebrionidae), langostas (Ortóptera), matapiojos (Odonata), mariposas nocturnas (Noctuidae), abejas y avispas varias (Hymenoptera) y frutas de Maitén.

Adicionalmente hay varias reseñas que hacen alusión de que es un ave cazadora de pequeños roedores y aves e.g., Gigoux (1928a), Housse (1945), Goodall et al. (1957), Martinez del Río (1992), Ridgely y Tudor (1994), Nuñez (1995), Cofre y Vilina (1999), lo cual puede ser algo engañoso y al parecer son solo acciones oportunistas, pero llaman la atención. En el área de estudio y en otras áreas del país nunca se observó a los adultos capturando o consumiendo batracios, roedores o consumiendo huevos de otras aves, pero si frutas de Maitén y pichones de otras aves. Sus principales presas eran lagartijas, orugas de Lepidópteros y arañas. Pero al parecer las menciones en su dieta como huevos, aves pequeñas y roedores son más secundarias o como subproducto debido a que su hábito principal de forrajeo parece ser en el suelo o entre ramas o captura al vuelo desde una percha. En el periodo post reproductivo su mayor cantidad 
de presas eran insectos terrestres y alguna que otra lagartija u araña, aunque en el invierno del 2012 un individuo se mantuvo unos tres meses cerca de unos panales de abejas melíferas, capturando al vuelo y luego consumiendo las abejas (Apis mellifera).

B) Dieta a los pichones: Se observó que ambos adultos alimentaban a los polluelos, en los tres a cuatro primeros días después de eclosionar parecía ser el que se presume el macho era el que traía alimento con más frecuencia, luego eran ambos con una frecuencia similar. Aunque se observó que la hembra capturaba sus presas más cerca del nido y el macho iba a más de 300-400 m del lugar. Dentro de los alimentos traídos a los pichones la gran mayoría eran lagartijas secundado por larvas de Lepidópteros ya sea orugas urticantes o suaves, una buena cantidad de arañas principalmente Arañas Pollito Grammostola rosea (Tabla 4). En la parte más tardía de los pichones, parecía ser el macho el que traía las presas más grandes, e.g., polluelos de aves y lagartijas grandes. Ocasionalmente, cuando uno de los adultos encontraba que los polluelos estaban ya saciados o cuando uno se acercaba al nido los adultos escondían sus presas, dejándolas entre la bifurcación de alguna rama de Espino u otro árbol/arbusto de ramas gruesas. En una ocasión el macho escondió su presa entre las ramas una lagartija, la cual pesó $11 \mathrm{~g}$ y la edad de los pichones era de 12-13 días (17,1\% del peso promedio de los polluelos de esa edad). El 59,6\% de la dieta a los pichones correspondía a pequeños vertebrados (ver Tabla 4) dentro de lo cual llama la atención que hay un cierto número de aves en la dieta a los polluelos. Las presas de aves capturadas no había aves adultas, todas eran polluelos con muy pocas plumas en el cuerpo y no se observó ningún polluelo ya crecido. Dentro de las especies traídas al nido se identificaron pichones de las siguientes especies: Cuculí Columbina picui, Diuca Diuca diuca, Loica Sturnella loyca, Fio-Fío Elaenia albiceps, y Chincol Zonotrichia capensis. Al menos dos especies encontradas en la dieta, la Loica y el Chincol, nidifican en el suelo y concuerda con sus hábitos de captura de presas (ver arriba). Sin embargo, la Cuculí, la Diuca, el Fio-Fío y algunas veces el Chincol, son arbóreos en su nidificación lo que también podría implicar búsqueda de alimento en pequeños arbustos. Aunque es más posible de haber localizado los nidos y los movimientos de los pichones desde alguna percha de acecho y luego capturar los pichones. Cacería desde las perchas de acecho es su modo de operar más común, después de buscar presas desde el suelo. Dentro de los vertebrados consumidos había al menos tres

Tabla 3. Cronología de eventos en el desarrollo del Mero (Agriornis lividus) en sector San Manuel, Melipilla, Chile.

\begin{tabular}{lc}
\hline Evento & Días de edad \\
\hline Cañones de las alas emergen & $4-5$ \\
Cañones del ala abren vaina, plumas emergiendo & $9-10$ \\
Cañones de la cola visibles, emergiendo & $5-6$ \\
Cañones de la cola abren vaina, plumas emergiendo & 10 \\
Plumas corporales abriendo vaina emergiendo & $8-9$ \\
Plumas corporales ya definidas, con muy poco plumón & $15-16$ \\
Línea del ojo comenzando a abrirse & $4-5$ \\
Abren los ojos completamente & $9-10$ \\
Diente de huevo desaparece & $13-18$ \\
Termina el empollado continuo & $7-8$ \\
Empollado parcial & $8-15$ \\
Activos al acercarse & $16-19$ \\
Salen del nido & $19-20$ \\
\hline
\end{tabular}


Tabla 4. Presas $(n=282)$ llevadas por los adultos a los polluelos de Mero Agriornis lividus, en el sector San Manuel, Melipilla

\begin{tabular}{|c|c|c|c|}
\hline Taxon & $\mathbf{n}$ & & $\%$ \\
\hline \multicolumn{4}{|l|}{ VERTEBRADOS (168) } \\
\hline Reptilia & & 159 & $56,4 \%$ \\
\hline Lagartigas (Liolaemus sp.) & 159 & & \\
\hline Aves & & 9 & $3,2 \%$ \\
\hline Passeriformes & 7 & & \\
\hline Columbiformes & 2 & & \\
\hline \multicolumn{4}{|l|}{ INVERTEBRADOS (114) } \\
\hline Arachnidae & & 26 & $9,2 \%$ \\
\hline Arañas peludas (Theraphosidae) & 24 & & \\
\hline Arañas pequeñas & 2 & & \\
\hline Lepidoptera & & 72 & $25,5 \%$ \\
\hline Orugas urticantes & 37 & & \\
\hline Orugas suaves & 34 & & \\
\hline Mariposas adultas & 1 & & \\
\hline Coleoptera & 12 & 12 & $4,2 \%$ \\
\hline Carabidae & 6 & & \\
\hline Larvas coleoptera & 6 & & \\
\hline Hymenoptera & 1 & 1 & $0,35 \%$ \\
\hline Cicadidae & 2 & 2 & $0,71 \%$ \\
\hline \multirow[t]{2}{*}{ Plecoptera } & 1 & 1 & $0,35 \%$ \\
\hline & 282 & & $100 \%$ \\
\hline
\end{tabular}

especies de lagartijas Liolaemus sp. muchos de los cuales eran juveniles, pero un número no menor eran de adultos. Las lagartijas más grandes eran adultos de Lagartija Chilena Liolaemus chilensis (una pesó $11 \mathrm{~g}$ ), secundada por Liolaemus tenuis. Los pichones de Cuculí que eran los polluelos más grandes que trajeron a los polluelos, estos los despresaba y traía por partes al nido. Después de alimentar los polluelos ambos adultos tomaban los sacos fecales y los depositaban a unos 10-15 m del nido, aunque dentro de los primeros días de edad de los polluelos, los adultos consumían los sacos fecales. El Mero al capturar sus presas, ya sea un invertebrado o vertebrado, las golpeaba, en una rama, en una piedra o en el suelo, asegurando la muerte de la presa.

Entre los comportamientos diferentes con otras aves paseriformes es que en el reclamo de los pichones por comida hacia los padres era silencioso la mayor parte del tiempo. Al llegar uno de los adultos al nido la solicitud de los polluelos era más visual que auditiva. Al llegar al nido muchas veces el adulto emitía un sonido en seco de una sola nota, sonaba algo como "tick" y los polluelos solo abrían la gran boca y ningún sonido era emitido por parte de los polluelos. Desde la edad de 8 a los 15 días, si los pichones no habían sido alimentados, y solo cuando acercaba uno de los adultos estos emitían un sonido algo como pick y repetido muchas veces, este era de alta frecuencia y casi inaudible. Esto coincidía con el principio del empollamiento parcial. El color del forro de la boca al centro era de un color amarillo anaranjado, los bordes amarillosos y la comisura un blanco opaco amarilloso de colores fuertes y atractivos. Con la gran 
boca abierta y silenciosos solo esperaban su comida. El adulto para decidir a cuál polluelo le pasaba la presa, este colocaba la presa en las diferentes bocas y al final se lo entregaba al polluelo que más pujaba por sus deseos de ser alimentado.

La diferencia que hay entre la dieta de los polluelos y la de los adultos en época post reproductiva es que la de los polluelos es menos variada y consistía en un $82 \%$ entre lagartijas y orugas (Tabla 4). Los adultos en la época post reproductiva consumían una gran variedad de presas variando desde insectos, caracoles, lepidópteros, pequeños vertebrados, hasta frutas (ver arriba). Pero su dieta principal eran presas del suelo ya sean insectos o caracoles.

\section{Mortalidad}

De 43 huevos de 12 nidos seguidos con detalles, la mayor mortalidad 14 (32,5\%) ocurrió en el estado de huevo, de los 14 huevos: 3 fueron depredados por algún tipo de ave (probablemente un Tordo (Curaeus curaeus) había un grupo de estos que compartía parte del territorio y había constantes encuentros agresivos, los huevos tenían perforaciones hechas por un pico y huecos hechos por un roedor (probablemente hechos por el roedor una vez rotos), 6 huevos abandonados por exceso de lluvia, y 5 no eclosionaron, probablemente por infertilidad o muerte de los embriones a temprana edad. Eclosionaron $29(67,4 \%)$ y de estos hubo una mortalidad de $6(20,6 \%)$ pichones. Tres pichones de 9-10 días fueron depredados por un Zorro Chilla (Dusicyon griseus) y tres pichones de una edad de 14-15 días, por un depredador aéreo desconocido ya sea Tiuques Milvago chimango o Chunchos Glaucidium nana ambos presentes en las cercanías del nido al momento. De los 29 pichones que eclosionaron 23 (79,3\%) salieron del nido exitosamente. Con un éxito reproductivo total del 53,4\%. Skutch (1976) reporta que para aves altriciales con nidos de taza abierta en la zona templada del hemisferio norte tienen un éxito reproductivo del $45,9 \%$, lo encontrado en este estudio 53,4\% es considerablemente más alto. El éxito reproductivo total para el Mero es alto comparado con otras especies que nidifican en nidos de taza abierta en la misma área por ejemplo la Diuca $31,7 \%$, la Tenca 26,3\%, el Diucón 43,2 \%, el Zorzal 40,8\% (Marín, 2011, 2012, 2013, 2015). Aunque las dos primeras especies la Diuca y la Tenca, su bajo éxito reproductivo está ligado a la destrucción de huevos y parasitismo por parte del Mirlo (Molothrus bonariensis) que es algo relativamente reciente ya que el Mirlo tiene solo alrededor de 100 años en el área (Marín, 2000). En cambio, el Diucón y el Zorzal se asemejan más a lo esperado para los nidos de especies altriciales de taza abierta. El alto éxito reproductivo del Mero, al menos en el área de estudio, puede deberse a que los nidos están en general muy protegidos, al ser construidos en plantas con alta densidad de espinas y adicionalmente es una especie grande y puede ser altamente agresiva contra algún depredador ya sea aéreo o terrestre. En ese contexto, el 29 de septiembre del 2010 observé una culebra de ca. 1,5-1,8 m tratando de acercarse a un nido con pichones, ambos adultos la atacaron sincronizadamente y esta se retiró del lugar. Adicionalmente en todos los nidos había constantes encuentros con Tordos y cuando estos estaban relativamente cerca de los nidos ambos adultos los atacaban hasta que se retiraran del lugar.

Dentro de los depredadores de huevos y pichones solo pude comprobar uno, el Zorro Chilla (Lycalopex griseus) que se comió los pichones de un nido localizado en una Puya en un lugar inclinado, otros tres quedaron como altamente sospechosos: el Tordo (Curaeus curaeus) que se trata de una especie conocida como depredadora de huevos y pichones e.g., Goodall et al. (1957), Marín (2013), estos se acercan a los nidos en grupos de 5-10 individuos, distraen los dueños y algún miembro del grupo roba o perfora los huevos. Adicionalmente, el Tiuque (Milvago chimango) y el Chuncho (Glaucidium nana) ambas especies se les encontraba frecuentemente en el área. 


\section{Movimientos}

$\mathrm{Al}$ área de estudio y en la zona cordillerana de las provincias centrales, la subespecie nominal, comienza a llegar en marzo-abril algunos años unos pocos individuos se mantienen en el área hasta julioagosto, luego si es un año favorable, algunos individuos se quedan a nidificar. Los años favorables se pueden definir como años con lluvias cerca de los 400-450 mm anuales o más, el promedio anual para el área es de $465 \mathrm{~mm}$. Las orugas de lepidópteras y las lagartijas son los dos ítems más importantes en la dieta de los polluelos (ver arriba) y creo que la cantidad de agua caída afecta la cantidad de huevos de orugas que eclosionan o que pueden sobrevivir. Adicionalmente, considero que la cantidad orugas de lepidópteras disponible para alimento es lo que puede determinar su nidificación.

Hay dos poblaciones bastante definidas divididas por la cordillera de la costa: la primera es de la zona costera hasta la cordillera de la costa de las zonas centro norte y la segunda es al este de la depresión central hasta los contrafuertes cordilleranos abarcando las zonas centro/sur hacia el extremo sur del continente. La población costera es un migrante austral parcial y estos se mueven solitarios o en pequeños números (he observado un máximo de cinco individuos en áreas cercanas, nunca juntos solo a cierta distancia) desplazándose hacia el norte por los valles bajos, planicies y zona costera, paran en áreas de preferencia con puyas y cactus, visitando las partes bajas de los valles de Huasco y de Copiapó, llegando como límite norte hasta la latitud de Caldera. En el área principal de estudio la llegada más temprana en los últimos 20 años fue el 24 de marzo del 2012 y 2021. Entre los meses de mayo a junio/julio es raro encontrarlo en el área. Aunque, hay años que uno que otro individuo se queda durante el invierno. En el área de estudio a finales de julio si el año es favorable se le puede encontrar en sus territorios y en agosto/ septiembre construyendo sus nidos. Individuos en la zona costera, en ambos extremos de su distribución por el norte (Región de Atacama) y por el sur (Región del Biobío) lugares o en áreas cercanas a Concepción y Angol, la especie es solo transitoria, por ejemplo, Lane (1897), Reed (1904) y Bullock (1929). La segunda población (que se distribuye en Argentina y Chile) es menos conocida y es más austral. Entre marzo y abril, se desplaza totalmente hacia el norte, principalmente por los pies cordilleranos. Individuos encontrados en la Región del Biobío al sur por los valles centrales y zonas costeras son solo individuos en transición migratoria ya sea de sur a norte a sus lugares de invernada o de norte a sur hacia sus lugares de reproducción. En las partes bajas de la Región de los Ríos, al sur es también una especie transitoria y se han avistado ejemplares en la zona norte de la región de Los Lagos, por ejemplo, en los alrededores de Puerto Varas, Puerto Montt y Chamiza y por las fechas de los especímenes de museo y avistamientos (e-bird 2021) es solo un ave de paso en dicha área.

\section{AGRADECIMIENTOS}

Se agradece a del Museo Nacional de Historia Natural, Santiago, Kimball Garrett de Los Angeles County Museum of Natural History, Los Angeles, EE.UU., Erika Theune, de Zapallar, Chile, J. Torres-Mura, Museo Nacional de Historia Natural, Santiago, Chile, Jeremiah Trimble, Museum of Comparative Zoology, (MCZ) Harvard University, EE.UU., Lloyd F. Kiff y Sam Sumida de la Western Foundation of Vertebrate Zoology, (WFVZ) Camarillo, California EE.UU., por el acceso a los especímenes a su cuidado. Se agradece la compañía de Christine Reed en las largas horas de observaciones. Rodrigo González quien me informo sobre los individuos observados en el sector La Parva, cordillera de Santiago. Se agradece a Jhoann Canto y a los revisores anónimos por mejorar este manuscrito. 


\section{REFERENCIAS BIBLIOGRÁFICAS}

ALBERT, F. 1899. Contribuciones al estudio de las aves chilenas. Familias: Phalacrocoracidae to Tyrannidae. Volume 2. Imprenta Cervantes, Santiago, Chile.

BARROS, R. 1921. Aves de la cordillera de Aconcagua. Revista Chilena de Historia Natural. 25: 167-192.

BARROS, R. 1961. Apuntes sobre el Diucón y los zorzales meros del centro. Revista Universitaria. 46: 155- 63.

BALDWIN, S. P., H. P. OBERHOLSER, y L.G. WORLEY. 1931. Measurements of birds. Scientific Publications of the Cleveland Museum Natural History. 2: 1-165.

BULLOCK, D. S. 1929. Aves observadas en los alrededores de Angol. Revista Chilena Historia Natural. 33: 171-211.

CASE, T. J. 1978. On the evolution and adaptative significance of postnatal growth rates in terrestrial vertebrates. Quarterly Review of Biology. 55: 243-282.

COFRÉ, H., y Y. A. VILINA. 1999. Depredación del Mero Agriornis livida (Passeriformes:Tyrannidae), sobre la Laucha de Pelo Largo, Akodon longipilis. Boletín Chileno de Ornitologia 6: 32-33.

CUNILL, P. 1970. Geografía de Chile. Editorial Universitaria, Santiago, Chile.

FITZPATRICK, J.W. 2004. Family Tyrannidae (Tyrant-Flycatchers) Pp. 170-463. In: del Hoyo, J., A. Elliott, y D.A. Christie (eds) Handbook of the Birds of the World. Vol 9. Cotingas to Pipits and Wagtails. Lynx Edicions, Barcelona, España.

FJELDSÅ, J. y N. KRABBE. 1990. Birds of the High Andes. Zoological Museum, University of Copenhagen and Apollo Books, Svendborg, Denmark.

FRASER, L. 1843. Sin título, pero son comentarios sobre una colección de aves llevadas al Reino Unido por el Sr. Bridges. Proceeding of the Zoological Society of London. 11:108-121.

GERMAIN, M. F. 1860. Notes upon the mode and place of nidification of some of the birds of Chili. Proc. Boston. Society of Natural History. 7: 308-316.

GIGOUX, E. 1928a. Los Agriornis. Revista Universitaria, Santiago. 13: 860-864

GIGOUX, E. 1928b. Aves de la Quebrada del León y alrededores. Revista Chilena de Historia Natural. 32: 144-148.

GOODALL, J.D., A.W. JOHNSON y R.A. PHILIPPI. 1957.Las aves de Chile su conocimiento y sus costumbres. Volumen 1. Platt Establecimientos Gráficos S.A., Buenos Aires, Argentina.

HELLMAYR, C. E. 1932. The Birds of Chile. Field Museum of Natural History Pub 308 Zoological Series Vol. XIX, Chicago. EE.UU.

HOUSSE, R. 1945. Las aves de Chile, en su clasificación moderna, su vida y costumbres. Ediciones de la Universidad de Chile, Santiago, Chile.

HUMPHREY, P.S., D. BRIDGE, P. W. REYNOLDS, y R. T. PETERSON. 1971. Preliminary Smithsonian Manual, Birds of Isla Grande de (Tierra del Fuego). University of Kansas Museum of Natural History. Kansas, EE. UU.

JOHNSON, A. W. 1967. The birds of Chile and adjacent regions of Argentina, Bolivia and Peru. Volume 2. Platt Establecimientos Gráficos S. A., Buenos Aires, Argentina.

KOVACS, C.J., O. KOVACS, Z. KOVACS, y C. M. KOVACS. 2005 Illustrated handbook of the Birds of Patagonia, Argentine Antarctica, and islands of the southern Atlantic. Museo Ornitológico Patagónico, El Bolsón, Rio Negro, Argentina.

KURODA, N. 1933. A collection of birds from Chile. Tori 8:128-147.

LANE, A. 1897. Field notes on the birds of Chili. With an introduction and remarks by P. L. Sclater. Ibis. 39: 8-51.

MARÍN, M. 2000. The Shiny Cowbird (Molothrus bonariensis) in Chile: Introduction or dispersion? Its host and parasitic trends. Ornitología Neotropical. 11:285-296. 
MARÍN, M. 2011.Sobre la biología reproductiva de la Diuca (Diuca diuca) en Chile central. Ornitología Neotropical. 22: $369-378$.

MARÍN, M. 2012.Historia natural y biología reproductiva de la Tenca (Mimus thenca) en Chile central. Boletín Museo Nacional Historia Natural, Santiago, Chile 61: 43-53.

MARÍN, M. 2013. Historia natural del Diucón (Xolmis pyrope) en Chile central, con énfasis en su biología reproductiva. Ornitología Neotropical 24:345-357.

MARÍN, M. 2015. Biología reproductiva e historia natural del Zorzal (Turdus faclklandii) en Chile central.Boletín Museo Nacional Historia Natural, Santiago, Chile 64: 81-99.

MARTINEZ DEL RIO, C. 1992.Great Shrike-tyrant predation on a Green-backed Firecrown. Wilson Bulletin 104:368369.

MILLIE, W. R. 1938. Las aves del valle del Huasco y sus alrededores (Provincia de Atacama). Revista Chilena de Historia Natural. 42: 181-205.

NUÑEZ, H. 1995. Ataque de Agriornis livida (Tyrannidae) a Passer domesticus (Ploceidae). Boletín Chileno de Ornitologia 2: 28.

PALMER, R. S. 1962. Handbook of North American birds. Volume 1. Yale Univ. Press., New Haven, Connecticut, EE.UU.

PHILIPPI, R. A. 1964.Catálogo de las aves de Chile con su distribución geográfica. Investigaciones Zoológicas Chilenas. 11:1-179.

REED, C.S. 1904. Las aves de la provincia de Concepción. Memoria de la exposición industrial y agrícola de Concepción de 1904. Imprenta Universitaria, Santiago, Chile.

REED, C.S. 1924. Notas biológicas referente a las aves chilenas. Revista Chilena de Historia Natural. 28:55-57.

RICKLEFS, R.E.1976. Growth rates of birds in the humid new world tropics. Ibis 118: 179-207.

RICKLEFS, R. E. 1983. Avian postnatal development. Pp. 1-83 en Farner, D. S., J. R. King, \& K. C. Parkes (eds). Avian biology. Volume 7. Academic Press, New York, EE. UU.

RIDGELY, R. S, y G. TUDOR. 1994. The birds of South America. Volume II. Univ. of Texas Press, Austin, Texas, EE. UU.

SKUTCH, A. F. 1976.Parent birds and their young. Univ. of Texas Press, Austin, Texas, EE. UU.

STARCK, J. M., y R. E. RICKLEFS. 1998. Avian growth rate data set. Pp. 381-415 en Starck, J. M. y R. E. Ricklefs, (eds). Avian growth and development, evolution within the altricial-precocial spectrum. Oxford Univ.Press, New York, EE. UU.

VUILLEMIER, F. 1994. Nesting, behavior, distribution, and speciation of Patagonian and Andean ground tyrants (Myiothereretes, Xolmis, Neoxolmis, Agriornis, and Muscisaxicola). Ornitología Neotropical. 5: 1-55.

ZOTTA, A. R. 1944. Lista sistemática de las aves Argentinas. Museo Argentino de Ciencias Naturales, Talleres Gráficos Tomás Palumbo, Buenos Aires Argentina.

Recibido: 29/oct/2021; Aceptado: 07/dic/2021; Administrado por Jhoann Canto H. 